\title{
Eops: Rastreo de cáncer de pulmón
}

\author{
Lung Cancer sreening
}

Karina Patané*

Patané K.Rastreo de cáncer de pulmón. Evid. actual. práct. ambul; 10(3): 92-94, may-jun.2007.

\section{Escenario}

Un cirujano torácico recibe en consulta a un paciente de 65 años cuyo hermano había sido intervenido quirúrgicamente hace cuatro meses, con diagnóstico de adenocarcinoma de pulmón, falleciendo un tiempo después. El mismo le expresa su preocupación, porque si bien abandonó el hábito tabáquico desde que empezó la enfermedad de su hermano, desea saber si es posible la detección temprana de ésta enfermedad con algún estudio diagnóstico.

Se ha estado postulando a la tomografía de baja dosis como posible método de rastreo de cáncer de pulmón en población de riesgo, pero no existe una aplicación masiva del método.

\section{Pregunta que generó el caso}

En pacientes tabaquistas, mayores de 60 años, con alto riesgo de cáncer de pulmón (población), ¿realizar un rastreo tomográfico anual (intervención) vs no realizarlo (comparación), mejora la sobrevida a 5 y 10 años? (resultado a valorar).

\section{Introducción al problema}

El cáncer de pulmón es la principal causa de muerte por cáncer en hombres y mujeres en los EE.UU. Se estima que en el año 2001 se han diagnosticado 169.500 nuevos casos, de los cuales 157.400 han muerto, es decir que la incidencia y la mortalidad a causa de la enfermedad casi se igualan. Más norteamericanos mueren de cáncer de pulmón cada año, que de cáncer de mama, próstata y colon juntos.

El tabaquismo es el agente causal principal de desarrollo de cáncer de pulmón (otras causas conocidas son: radiación, asbesto, polución ambiental, etc.). Entre fumadores el riesgo de enfermedad se incrementa en forma directamente proporcional con el número de cigarrillos/día y con el tiempo de fumador activo. Por lo tanto, aquellos que se inician más tempranamente en éste hábito tienen más posibilidades de desarrollar cáncer de pulmón y a edades más tempranas. Si bien el cese del tabaquismo es benéfico a cualquier edad, luego de 40 años de cese tabáquico, el riesgo sigue siendo mayor para los ex-fumadores, que para los no fumadores y los fumadores pasivos tienen un $30 \%$ más probabilidades de desarrollar cáncer que los no expuestos a humo del tabaco. Por otro lado, las mujeres tienen un riesgo mayor que los hombres de desarrollar cáncer de pulmón, con el mismo nivel de tabaquismo. La sobrevida global a 5 años es del 14\%. Considerando los estadíos, la sobrevida a 5 años para los Estadíos I es del $75 \%$, para los Estadíos II es del 35\% y para los Estadíos III es de sólo el 15\%. De ahí el esfuerzo en encontrar un método de rastreo que permita detectar más pacientes en Estadío I de la enfermedad, de modo de lograr un resultado favorable en la sobrevida.

Han sido utilizados con tal fín la citología de esputo, la radiología seriada y ambos métodos combinados, con poco impacto en la sobrevida. En estos últimos tiempos ha surgido interés acerca del uso de la tomografía computada de baja dosis, y algunos resultados parecen ser alentadores (ver artículo en página 77).

\section{Estrategia de búsqueda y resultados}

Se realizó una búsqueda en Medline empleando como palabras clave " screening" AND "lung cancer" AND " low dose CT". Se limitó la búsqueda a artículos en inglés, español o italiano, publicados en los últimos tres años, y preferentemente revisiones sistemáticas y meta-análisis del tema.

Con estos límites se obtuvo un solo artículo que reunía esas características:

Diederich,S; Wormanns, D; Heindel, W: Lung cancer screening with low dose CT, European Journal of Radiology 45(2003) 2-7.

Sin embargo, ampliando la búsqueda a trabajos publicados en el último año, aunque no fueran ECCAs o revisiones sistemáticas, hay al menos dos trabajos interesantes al respecto, el primero por el número de pacientes enrolados y el segundo por el análisis de costo efectividad del rastreo tomográfico en pacientes de riesgo:

1) ELCAP; Survival of patients with stage I lung cancer detected on CT screening, NENOL D MEI, Oct 2006; 355:1763-1771.

2) Kishor Ganti, A; Mulshine, J; Lung Cancer screening, The Oncologist, 2006;11:481-487.

\section{Resumen de la evidencia}

Diederich,S; Wormanns, D; Heindel, W: Lung cancer screening with low dose CT, European Journal of Radiology 45(2003) 2-7.

Éste artículo publicado en el 2003, revisa las investigaciones disponibles hasta el momento acerca de éste ítem. Resume al menos 4 trabajos, entre ellos los resultados preliminares del que discutiré abajo, todos con escaso número de pacientes (hasta ese momento) y todos observacionales. Con esos datos concluye que sólo un ensayo clínico puede responder el interrogante acerca del rastreo tomográfico en cáncer de pulmón. Cabe destacar que hace hincapié en todos los posibles sesgos de un método de rastreo: lead-time bias, lenght-time bias, sesgo del voluntario y sesgo de sobrediagnóstico (pacientes con cáncer de pulmón que mueren por otras causas y por lo tanto no alteran la mortalidad global de la enfermedad).

ELCAP; Survival of patients with stage I lung cancer detected on CT screening, NENOL D MEI, Oct 2006; 355:1763-1771.

\section{Objetivo: y Material y métodos: ver artículo pág. 77}

\section{Características de los participantes}

Línea de base

- 31567 participantes

- Mediana de la edad: 61 ( rango 40-85)

- Historia de tabaquismo: mediana 30 p/y

- Exposición laboral (asbesto, berilio, uranio o radón)

- Fumadores pasivos

Rastreo anual

- 21456 participantes.

- Mediana de la edad: 62 años( 41-86)

- Mediana de paquetes/año: 35. 


\section{$\underline{\text { Resultados }}$}

- Sobrevida a 10 años sin tener en cuenta el estadio: 80\% (IC $95 \% 74$ a 85 ).

- 412/484 eran estadio I: sobrevida poyectada a 10 años: $88 \%$ (IC 95\% 84-91).

- 347/412 tuvieron confirmación patológica de estadio I: sobrevida proyectada a 10 años: 94\%( IC 95\% 91-97).

Para establecer un parámetro de comparación de la efectividad del método, se compararon las tasas de detección basales y anuales del rastreo tomográfico con la mamografía, con éstos resultados:

- Tasa de detección basal vs mamografía: $1.3 \%$ vs 0.6 a $1 \%$.

- Tasa de detección anual vs mamografía: $0.3 \%$ vs 0.2 a 0.4 .

- La tasa de detección depende del perfil de riesgo de los sujetos a rastrear: a mayor riego mayor detección.

\section{Conclusiones de los autores:}

- La costo efectividad del método debe ser estimada de la siguiente manera: costo de la TAC/ costo del tratamiento oncológico en estadíos avanzados.

- Siguiendo el razonamiento anterior: el rastreo de cáncer de pulmón es altamente costo efectivo.

(Nota: el estudio no evalúa expresamente la costo-efectividad de la política de rastreo)

Kishor Ganti, A; Mulshine, J; Lung Cancer screening, The Oncologist, 2006;11:481-487

En particular éste trabajo revisa la bibliografía y discute el anterior y otros trabajos publicados sobre rastreo tomográfico en cáncer de pulmón. Algunas conclusiones interesantes son:

- Si bien es deseable contar con ensayos clínicos que estudien el valor de la intervención (rastreo tomográfico) vs la práctica clínica habitual, un estudio observacional bien conducido puede ofrecer resultados muy válidos sobre la efectividad, así como los de un ECCA informan sobre eficacia.

- Si bien una de las críticas del rastreo tomográfico ha sido la alta tasa de falsos positivos, esto parece tener que ver con la técnica utilizada en el estudio: un trabajo publicado por la Mayo Clinic reportó una tasa de falsos positivos global del $96 \%$ vs el $23 \%$ del estudio anterior. Esto parece debido a que en el primero se utilizó una técnica de four- slice, mucho más sensible que el single slice utilizado en el ELCAP.

- Si bien el análisis de costo-efectividad no ha sido prolijo en el estudio del ELCAP, éste trabajo resume varios análisis de costo efectividad y costo- utilidad, muchos alentadores y otros no tanto, a saber: en un modelo simulado sobre los hallazgos de la Mayo Clinic el costo del rastreo para fumadores activos era de U\$S 116.300 por QALY* ganado, superando los dos millones de dólares por Qaly en ex fumadores, haciendo éste método de rastreo extremadamente caro. Sin embargo los cálculos estimados según datos del ELCAP y un estudio italiano de Pastorino, informan un costo de U\$S 2500 por año de vida ganado, y sólo se excede los U\$S 50000 del cociente de costo/efectividad si se produce un sobrediagnóstico del $50 \%$. Esto demuestra la enorme variabilidad que existe sobre el método y los costos de la intervención y las distintas formas e evluar efectividad $\mathrm{y} / \mathrm{o}$ utilidad.

- Por último, la dosis de radiación utilizada con la técnica helicoidal minimiza el riesgo de nuevos cánceres por radiación, dado que el monto de la misma es igual al de una radiografía simple de tórax de frente y perfil.

\section{Comentario crítico}

Definición de método de Rastreo: se refiere a la aplicación de un test a personas asintomáticas con el propósito de clasificarlas con respecto a su probabilidad de tener o no una enfermedad.

Se asume que la detección temprana, antes del comienzo de los síntomas, mejora el pronóstico (y la sobrevida) de éstos pacientes, debido a que el tratamiento temprano es más efectivo que el tardío.

Considero necesario analizar la tomografía de baja dosis desde distintas perspectivas:

- Características de la enfermedad

El cáncer de pulmón presenta las características necesarias para implementar un método de rastreo: representa una enfermedad seria, el tratamiento en estadios tempranos de la enfermedad mejora el pronóstico (si detectáramos a todos los pacientes en estadío I mejoraríamos la sobrevida notoriamente; hasta ahora ningún método logró éste resultado) y la prevalencia de la fase preclínica de la enfermedad es alta, sobre todo en pacientes de alto riesgo.

$1^{\circ}$ Conclusión: El cáncer de pulmón es una enfermedad con las características necesarias para implementar un método de rastreo.

\section{Características del método de rastreo ideal}

- Fácil de administrar.

- Genera mínima incomodidad.

- Disponible

- Económico: éste punto puede ser el más débil, por eso resulta útil analizarlo desde la perspectiva de la costo/ efectividad.

$2^{\circ}$ Conclusión: La tomografía computada parece reunir las características básicas de un método de rastreo adecuado.

\section{Condiciones a cumplir por un método de rastreo}

- Validez: considerada como su capacidad para categorizar correctamente enfermos y sanos. La tomografía computada parece ser un método válido, según éste trabajo para dicha categorización. No están expresados sin embargo en el trabajo los valores de sensibilidad del método para el rastreo anual y basal, así como tampoco los valores predictivos positivos y negativos y los correspondientes cocientes de probabilidad (Likelihood ratios o LR).

- Confiabilidad: los resultados son reproducibles.

- Metodología: preferiblemente ECCAs. Si bien éste estudio es descriptivo, parece bien conducido y el número de pacientes enrolados es altísimo.

$3^{\circ}$ Conclusión: no están claramente expresados en el trabajo todos los datos relevantes para evaluar la validez del método, aunque pueden estimarse (ver abajo).

\section{Posibles sesgos}

- Sesgo de voluntario: seguramente los voluntarios que ingresaron al protocolo presentan características diferentes a las de la población general, o tiene un cuidado excesivo de su salud y por lo tanto suelen tener mejor pronóstico, o por el contrario se enrolan por antecedentes familiares de neoplasia, lo cual hace que su pronóstico, dada la posible carga genética, sea desfavorable. 
- Sesgo de Anticipación*: debido a que no están expresadas la edad de muerte de los pacientes, no fue controlado éste sesgo en el trabajo y no es posible saber si la sobrevida a 5 y 10 años es un beneficio real.

- Sesgo de Duración*: es imposible de predecir y controlar el comportamiento biológico de la enfermedad. En aquellos pacientes en los cuales la fase preclínica es más corta, el rastreo adquiere poco valor. En éste trabajo, los 5 pacientes que fueron diagnosticados entre el rastreo basal y el anual pueden reflejar ésta circunstancia.

$4^{\circ}$ Conclusión: En ningún momento en el trabajo se describen los posibles sesgos y limitaciones, y parece ser susceptible de todos ellos.

\section{Cuáles son los resultados?}

- No están expresados los valores predictivos positivos y negativos del test, tanto en el rastreo basal como en el anual. - Considerando los datos expresados en el estudio, los resultados podrían ser los siguientes

\section{Rastreo basal}

\begin{tabular}{|l|l|l|}
\hline Test + & Enf. presente & Enf. ausente \\
\hline Test - & 405 & 3781 \\
& 5 & 27376 \\
\hline
\end{tabular}

\section{Especificidad: VN/ VN+FP=88\% Sensibilidad: VP/ VP+FN=98.8\% VPP: VP/ VP+FP= $10 \%$ VPN: VN/VN+FN= $99 \%$}

\section{Rastreo anual}

\begin{tabular}{l|l|l|}
\hline Test + & Enf. presente & Enf. ausente \\
\hline Test - & 0 & 1386 \\
& 0 & 25996 \\
\hline
\end{tabular}

\section{Especificidad: VN/ VN+FP=95\% \\ Sensibilidad: VP/ VP+FN=100\% \\ VPP: VP/ VP+FP=5\% \\ VPN: VN/VN+FN=100\% \\ LR $+=$ sens $/ 1$-esp $=20$ \\ LR - = 1-sens/ esp $=0$}

\section{Conclusiones:}

El rastreo de cáncer de pulmón con tomografía computada de baja dosis parece un método útil para la detección temprana y mejora del pronóstico de éstos pacientes. En la práctica diaria la utilización de éste método debe incluir una clara explicación al paciente acerca de los posibles falsos positivos del método y por ende las intervenciones diagnósticas y sus posibles complicaciones. Falta definir claramente cuál es la población blanco, en quienes sería más costo- efectivo el procedimiento y más estudios de costo efectividad, lo cual no está correctamente expresado en el trabajo.

Como expuse anteriormente, para sumar certeza a las conclusiones, y siendo el cáncer de pulmón una de las enfermedades en la cuales ha sido muy costoso encontrar un método de rastreo útil, sería necesario controlar el sesgo de anticipación (o lead time bias), expresando las edades de muerte de los pacientes para evaluar el beneficio real de la intervención.

Las características operativas del test sugieren que, para el rastreo de cáncer de pulmón en pacientes de alto riesgo, la tomografía computada de baja dosis tiene una adecuada capacidad de discriminación y un elevado valor predictivo negativo, aunque su tasa de falsos positivos y los costos globales asociados determinan la necesidad de realizar más y mejores estudios que evalúen la costo efectividad de la intervención antes de poder recomendarla.

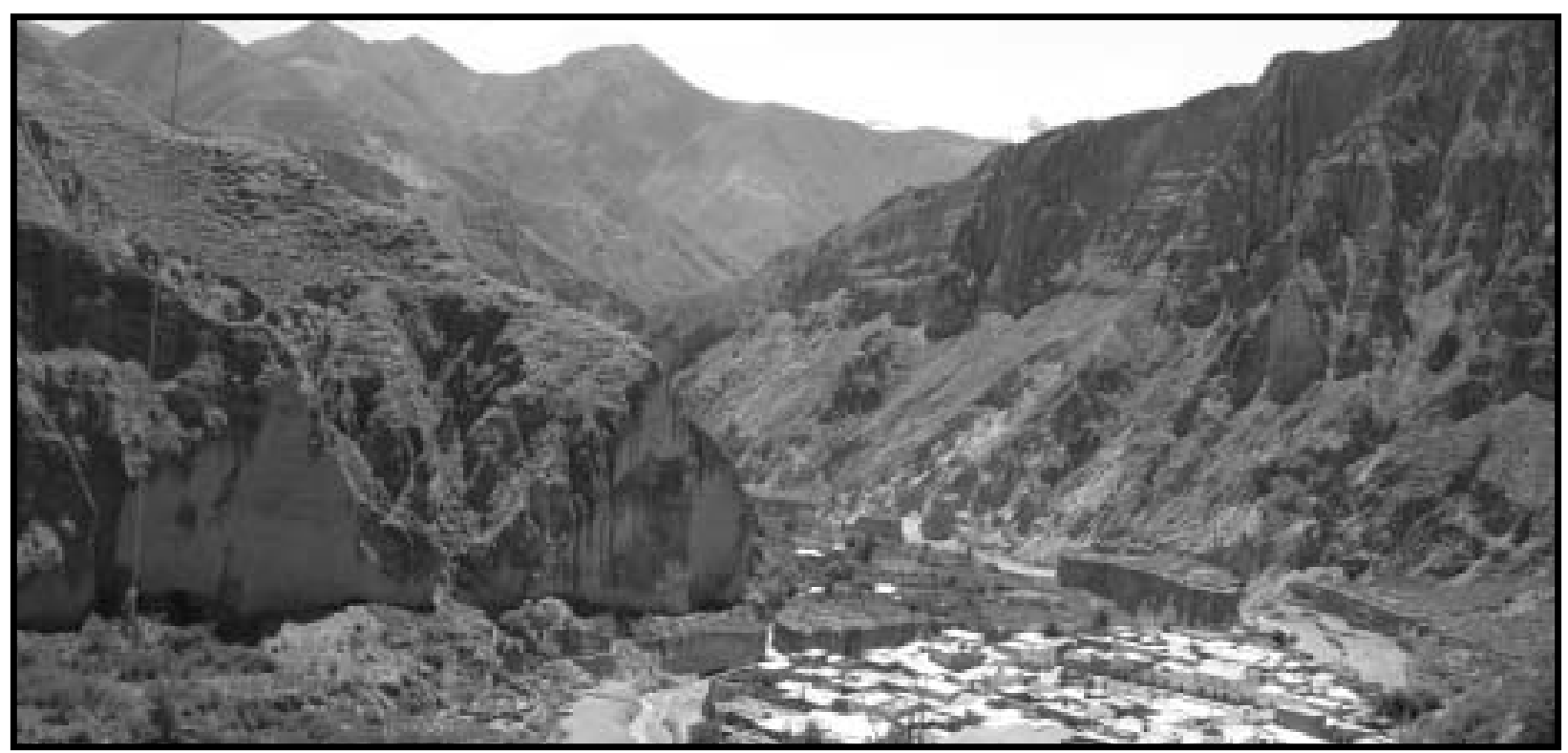

Iruya, Salta, Argentina. Cortesía: Fotos: Guadalupe Alessia. 